Research Article

\title{
Gender Variations in the Oral Microbiomes of Elderly Patients with Initial Periodontitis
}

\author{
Jie Zhao, ${ }^{1}$ Ying-Hui Zhou, ${ }^{2}$ Ya-Qiong Zhao, ${ }^{1}$ Yao Feng, ${ }^{1}$ Fei Yan $\mathbb{D}^{,}{ }^{3}$ Zheng-Rong Gao, \\ Qin Ye, ${ }^{1}$ Yun Chen, ${ }^{1}$ Qiong Liu, ${ }^{1}$ Li Tan, ${ }^{1}$ Shao-Hui Zhang, ${ }^{1}$ Jing Hu, ${ }^{1}$ \\ Marie Aimee Dusenge, ${ }^{1}$ Yun-Zhi Feng $\mathbb{D}^{1},{ }^{1}$ and Yue Guo $\mathbb{D}^{1}$ \\ ${ }^{1}$ Department of Stomatology, The Second Xiangya Hospital of Central South University, Changsha, 410011 Hunan, China \\ ${ }^{2}$ National Clinical Research Center for Metabolic Diseases, Hunan Provincial Key Laboratory of Metabolic Bone Diseases, \\ Department of Metabolism and Endocrinology, The Second Xiangya Hospital of Central South University, Changsha, \\ 410011 Hunan, China \\ ${ }^{3}$ Hunan Key Laboratory of Oral Health Research \& Hunan 3D Printing Engineering Research Center of Oral Care \& Hunan \\ Clinical Research Center of Oral Major Diseases and Oral Health \& Xiangya Stomatological Hospital \& Xiangya School \\ of Stomatology, Central South University, Changsha 410008, China
}

Correspondence should be addressed to Yun-Zhi Feng; fengyunzhi001@csu.edu.cn and Yue Guo; guoyue@csu.edu.cn

Received 2 June 2021; Accepted 30 October 2021; Published 23 November 2021

Academic Editor: E. Xiao

Copyright (C) 2021 Jie Zhao et al. This is an open access article distributed under the Creative Commons Attribution License, which permits unrestricted use, distribution, and reproduction in any medium, provided the original work is properly cited.

Periodontitis is a globally prevalent disease that imposes a functional and aesthetic burden on patients. The oral microbiome influences human health. The aim of this study was at assessing gender variation in the subgingival bacterial microbiome of elderly patients with initial periodontitis and to determine the causes of this variation. Twelve males and twenty females (range 50-68 years old) with initial periodontitis provided subgingival plaque samples. 16S rRNA gene sequencing, QIIMEbased data processing, and statistical analyses were carried out using several different analytical approaches to detect differences in the oral microbiome between the two groups. Males had higher Chao1 index, observed species, and phylogenetic diversity whole tree values than females. Analysis of $\beta$-diversity indicated that the samples were reasonably divided by the gender. The linear discriminant analysis effect size showed that the most representative biomarkers were the genus Haemophilus in males, whereas the dominant bacteria in females were Campylobacter. Kyoto Encyclopedia of Genes and Genomes analysis showed that predicting changes in the female oral microbiota may be related to the immune system and immune system diseases are the main factor in males. These data suggest that gender may be a differentiating factor in the microbial composition of subgingival plaques in elderly patients with initial periodontitis. These results could deepen our understanding of the role of gender in the oral microbiota present during initial periodontitis.

\section{Introduction}

The human oral cavity is composed of unique niches colonized by a variety of microorganisms, including bacteria, fungi, viruses, and archaea, which create a rich microbial community known as the oral microbiome [1]. These communities of microbes are highly dynamic and responsible for various functions that can both maintain and deplete oral health. Oral microbiomes often exist in a homeostatic equilibrium with the host, but under certain conditions, this equilibrium is disturbed. Oral microbiome dysbiosis can cause a wide range of systemic illnesses, including diabetes [2], cancer [3], Alzheimer disease [4], rheumatoid arthritis [5], and cardiovascular disease [6]. For example, a higher pancreatic cancer risk has been associated with the enrichment of Porphyromonas gingivalis and Aggregatibacter actinomycetemcomitans [7]. Furthermore, oral $P$. gingivalis infection in mice results in brain colonization of an Alzheimer's disease model and increased production of $\mathrm{A} \beta 1-42$, a component of amyloid plaques [8]. Oral microbiome 
dysbiosis also directly affects oral health, potentially causing periodontitis and dental caries, two of the most prevalent and typical microbially induced disorders worldwide. Such periodontal disease will also affect systemic health, including the immune system [9] and digestive system [10].

Age correlates with the severity of periodontal disease and dental caries. Because of the decline in immune system function and low salivary flow rates brought on by the natural advancement of age, there is a clinical increase in the prevalence and severity of periodontitis and dental caries in older adults. Age is also an important factor in oral microbiome dysbiosis. Rodenburg et al. found that the prevalence of subjects colonized by $A$. actinomycetemcomitans appeared to be age related, as it decreased with increasing age [11]. In addition, the incidence and severity seem to have a gender predilection with the incidence and severity of periodontal disease appearing to be greater in males than in females $[12,13]$. Lira-Junior et al. found that male gender presented higher counts of 16 bacteria in saliva than female gender, which may be related to the slightly worse periodontal condition in male than in female [13]. Furthermore, the NHANES III survey has demonstrated, in most age groups, a higher prevalence and greater extent of attachment loss, as well as a higher prevalence of periodontitis and deeper probing depths in males compared to females [14]. However, females have been shown to be more susceptible to caries [15]. Thus, gender can logically be assumed to be an important factor affecting the oral microbiome.

The immunological response produced by the oral microbiome is important and complex. Tissue homeostasis is maintained by innate immunity, which prevents destruction of the periodontal tissue. Severe periodontitis is characterized by neutropenia, agranulocytosis, neutrophil adhesion, deficiencies in chemotaxis, and diseases affecting lysosomal degranulation [16]. The cellular infiltrate in human gingivitis is primarily composed of $\mathrm{T}$ helper (Th) cells. The phenotypes of Th cells are directed by phagocytic antigen-presenting cells, including dendritic cells (DCs) and Langerhans cells [17]. The immune response in the gingiva, during an undefined transition, switches from neutrophil recruitment and activation to pathogenic bacteria to chronic infiltration of $\mathrm{T}$ and $\mathrm{B}$ cells and plasma cells [18] that induces vascular prolifera and the destruction of connective tissue. At the same time, sex steroids are related to the immune system. Youssef and Stashenko indicated that estrogen directly stimulates IL-1 production by macrophages, leading to heightened immune responses and the resistance of females to disseminating dentoalveolar infections [19]. In contrast, androgen has been reported to play a suppressive role in the immune response and to act as a potential promoter of tumor growth and protector from autoimmune diseases [20]. Androgen-deficient male C57BL/6 mice were shown to be significantly more susceptible to endotoxic shock, possibly due to the significantly higher expression of surface TLR4 on macrophages [21].

Therefore, the aim of the present study was to assess gender variation in the subgingival bacteria microbiome of elderly patients with initial periodontitis and determine the causes of this variation. Specifically, we examined whether bacterial taxa were differentially abundant, whether gender variation exists in the $\alpha$ - and $\beta$-diversity using highthroughput sequencing data, and whether the variation is related to the immune system.

\section{Materials and Methods}

2.1. Study Population and Sample Data. All participants were recruited from The Second Xiangya Hospital of Central South University in Changsha, China. The Ethics Committee of The Second Xiangya Hospital of Central South University provided ethics and regulatory approval. Verbal and written informed consent were obtained from all participants. The clinical trial registration number is ChiCTR2100046828.

The participants included in this study were 50 to 68 years old [22-24], with $\geq 15$ teeth present and at least one natural tooth in all six sections. Exclusion criteria included smoking, infection, trauma or recent surgery, pregnancy, systemic disease (e.g., heart failure, autoimmune disease, musculoskeletal disorders, and malignancy), the use of antibiotics or immunomodulators in the previous 30 days, periodontal treatment in the previous 6 months, or oral disease (e.g., oral lichen planus, ulcer, oral leukoplakia, and pharyngitis). Questionnaires were used to obtain data from all patients who agreed to serve as subgingival plaque donors. We enrolled 32 patients with initial (stage I) periodontitis, including 12 males and 20 females. Before collecting samples, sites were air dried. The stage of periodontal disease was determined according to the 2017 World Workshop on the Classification of Periodontal and Peri-Implant Diseases and Conditions "Staging and grading of periodontitis: Framework and proposal of a new classification and case definition" [25]. The clinical attachment level (CAL) of the most severe site was recorded for each tooth; 1-2 $\mathrm{mm}$ was defined as stage I, 3$4 \mathrm{~mm}$ as stage II, and $\geq 5 \mathrm{~mm}$ as stage III. The number of missing teeth was also staged, with no missing teeth indicating stages I and II, $\leq 4$ teeth missing stage III, and $\geq 5$ teeth missing stage IV. Notably, the reasons for the missing teeth were not considered. The complexity of management was evaluated by reclassifying stage II patients as stage III if the maximum probing pocket depth (PPD) was $\geq 6 \mathrm{~mm}$, and stage III patients as stage IV if $<20$ teeth or 10 opposing pairs remained. The subgingival plaques of the four first molars were scraped at the bottom of the periodontal pocket using a sterile Gracey scraper. The samples were transferred to PBS, immediately frozen, and maintained at $-80^{\circ} \mathrm{C}$.

2.2. DNA Extraction and Sequencing of the $16 S$ rRNA V3-V4 Region. Based on the manufacturer's instructions, total genomic DNA was extracted using a DNA Extraction Kit (DNeasy PowerSoil Kit, QIAGEN). The extracted DNA was quantified using a NanoDrop 2000 spectrophotometer (Thermo Fisher Scientific, Waltham, MA, USA) and the quality confirmed by agarose gel electrophoresis. The universal PCR primers $343 \mathrm{~F}$ ( $5^{\prime}$-TACGGRAGGCAGCAG-3') and $798 \mathrm{R}$ ( $5^{\prime}$-AGGGTATCTAATCCT-3') were designed to amplify the V3-V4 variable regions of bacterial 16S rRNA genes. 16S rRNA gene sequencing was completed by 
OEBiotech (Shanghai, China). PCR was performed using the following program: initial denaturation at $94^{\circ} \mathrm{C}$ for $5 \mathrm{~min}, 26$ cycles of denaturation at $94^{\circ} \mathrm{C}$ for $30 \mathrm{~s}$, annealing at $56^{\circ} \mathrm{C}$ for $30 \mathrm{~s}$, elongation at $72^{\circ} \mathrm{C}$ for $20 \mathrm{~s}$, and a final extension at $72^{\circ} \mathrm{C}$ for 5 min. Amplicons were purified using AMPure XP beads (Agencourt, Beckman Coulter, USA) and quantified using the Qubit dsDNA Assay Kit (Life Technologies, USA) following the manufacturers' instructions. Purified amplicons were pooled for sequencing. The raw reads were generated by Illumina MiSeq (V1.9.1), and the sequences were processed and analyzed in QIIME (v1.9.1). Briefly, the effective sequences were clustered to the same OTUs with $\geq 97 \%$ identity using VSEARCH (v.2.4.2) [26]. Next, the annotation information of representative sequences of each OTU was analyzed using the RDP classifier Naive Bayesian method [27] and the SILVA database.

2.3. Bioinformatics and Statistical Analysis. The $\alpha$-diversity indices (Chao, Shannon, Simpson, Good's coverage, and phylogenetic diversity (PD) index) were calculated at $97 \%$ identity by the Wilcoxon rank-sum test. Samples from different groups were compared by Student's $t$-test and the Kruskal-Wallis test. Beta diversity analysis was performed by principal coordinates analysis (PCoA) based on unweighted UniFrac distances at the OTU level. Principal component analysis (PCA) was also conducted. The analysis of nonmetric multidimensional scaling (NMDS) based on weighted UniFrac distances was conducted to compare different groups. The relative abundance of predominant bacteria was compared between different groups using the Wilcoxon rank-sum test. The core microbiome was defined at the species level using a Venn diagram. We performed linear discriminant analysis (LDA) of effect size (LEfSe) to define biomarkers in the four groups. The logarithmic LDA score threshold for distinguishing features was 2.0. The functional content from the16S rRNA gene sequences was predicted using phylogenetic investigation of communities by reconstruction of unobserved states (PICRUSt) software [28] by linking taxonomic information to the Kyoto Encyclopedia of Genes and Genomes (KEGG) annotations of the reference genome. Differences were considered significant when $P<0.05$. SPSS 25.0 software was used for statistical analyses.

\section{Results}

3.1. Characteristics of Selected Patients and OTU Basic Analysis. Patient age $(n=32)$ ranged from 50 to 68 years. The male patients had a mean age of 54 years (range 5160 years) and female patients 58.6 years (range $50-68$ years). There was no statistically significant difference in age between male and female $(P>0.05$, Table 1$)$. The Venn diagram of differences in the OTUs (Figure 1(a)) showed that males and females had 3477 common OTUs. However, 1642 OTUs were higher in females and 1165 in males.

3.2. Diversity Analyses. Alpha diversity reflects the abundance and diversity of microbial communities. The Chao 1 index and observed species were used to calculate commu-
TABle 1: Age information of the enrolled participants.

\begin{tabular}{lcc}
\hline Group & Number & Age (year) \\
\hline Group 1 & 12 & $54.00 \pm 3.54$ \\
Group 2 & 20 & $58.55 \pm 7.45$ \\
$P$ & & $>0.05$ \\
\hline
\end{tabular}

nity richness, whereas the Shannon and Simpson indices were able to evaluate community diversity. Compared to females, males had a significantly higher number and/or diversity of taxa based on the Chaol index $(P<0.01$, Figure $1(\mathrm{~b}))$ and observed species $(P<0.01$, Figure $1(\mathrm{c}))$. No significant difference was apparent between the two groups in the Shannon (6.37 versus 6.56, Figure 1(d)) and Simpson (0.96 versus 0.97, Figure 1(e)) indices. Higher Good's coverage index values were associated with a higher probability of species being measured in the sample. Although Good's coverage was significantly different between the two groups $(P<0.01)$, both groups reached 0.99 for the depth of sequence representing the majority of bacterial species in the plaque samples. The PD whole tree index reflects the relationships of species within the community and was found to decrease in females compared to males (Figure 1(g)).

Beta diversity refers to visualization of the differences in the diversity of microbiota in the two groups. The PCA (Figure 2(a)) and PCoA (Figure 2(b)) indicated the extent of the difference in the spatial distance, especially in PCoA analysis based on unweighted UniFrac distance. In NMDS analysis based on the binary Jaccard distance, we found a significant spatial distance and value of stress $<0.2$, representing certain explanatory significance in the twodimensional point graph (Figure 2(c)). The male and female groups were far apart (Figure 2), indicating that the oral microbiotas in elderly with initial periodontitis vary by gender.

3.3. Composition of the Oral Microbiota. The relative abundance of the top 15 bacteria was assessed at the phylum level (Figure 3(a)) and genus level (Figure 3(b)). Multivariate analysis estimated further differences between the two groups and identified bacteria that differed significantly between them. A boxplot shows the top 3 different species at the phylum (Figure 3(c)) and top 10 different species at the genus (Figure 3(d)) levels. Compared to females, the relative abundance of cyanobacteria at the phylum level increased in males, whereas the relative abundance of Epsilonbacteraeota and Fibrobacteres was decreased $(P<0.05$; Figure 3(c)). At the genus level, females had higher relative abundance of Campylobacter than males $(P<0.05)$. However, the relative abundance of Haemophilus, Bacteroides, Prevotellaceae_UCG-001, Clade_Ia, Acinetobacter, Ruminococcus_1, Prevotellaceae_NK3B31_group, Ruminococcaceae_ UCG-005, and Parabacteroides was lower in females than in males $(P<0.05$; Figure $3(\mathrm{~d}))$. Figure 4 shows the different species between the two groups in the form of a heat map.

We conducted LEfSe to identify the differential bacterial composition between the male and female patients and 

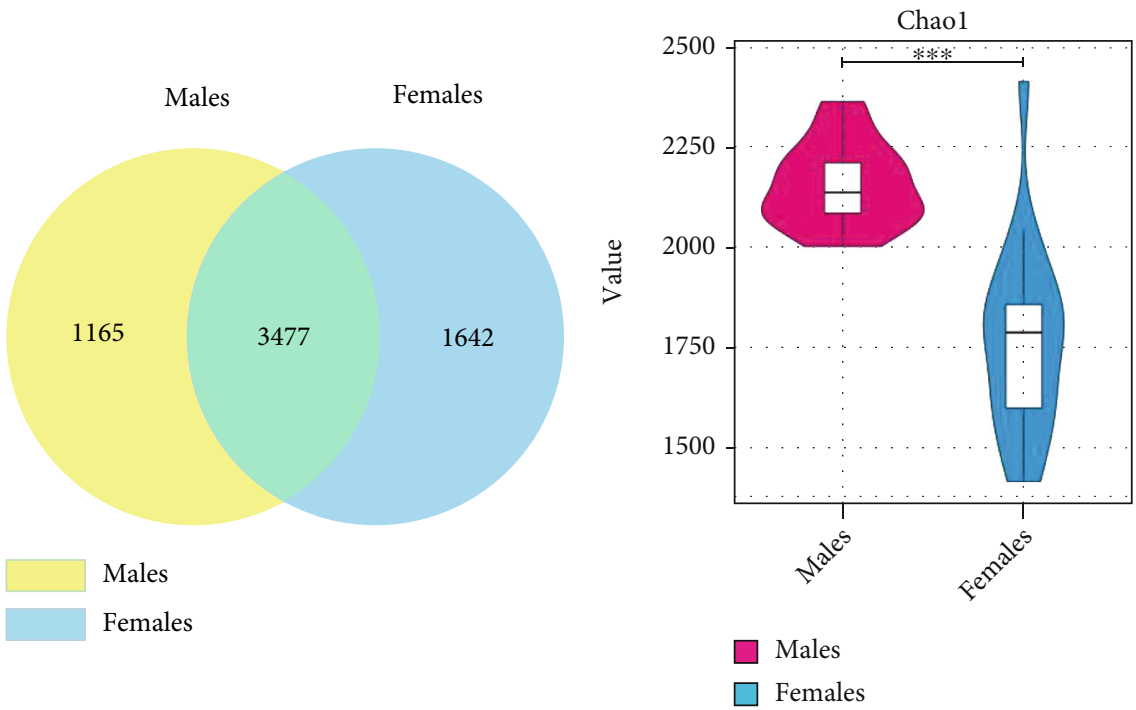

(a)

(b)

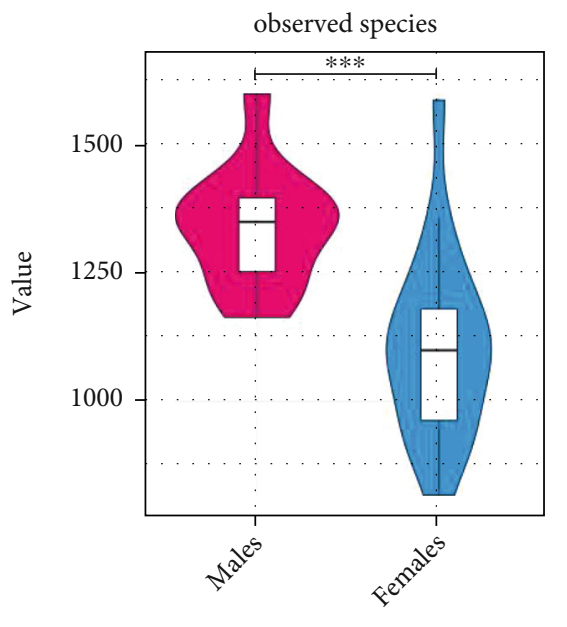

Males

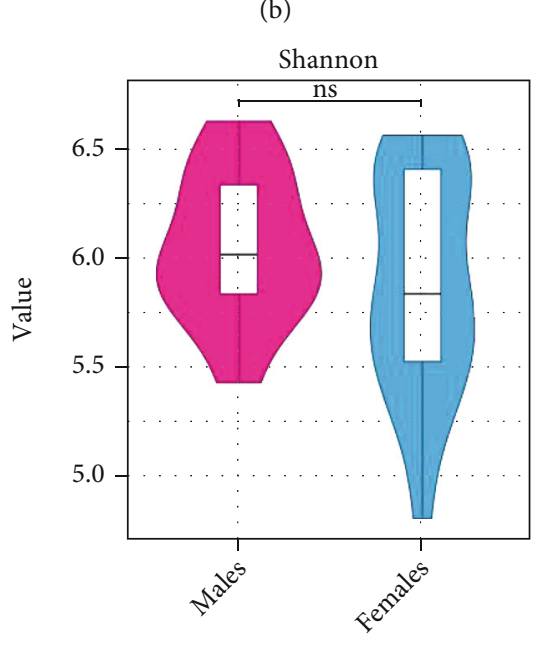

$\square$ Females

$\square$ Males

$\square$ Females

(c)

(d)

Figure 1: Continued. 


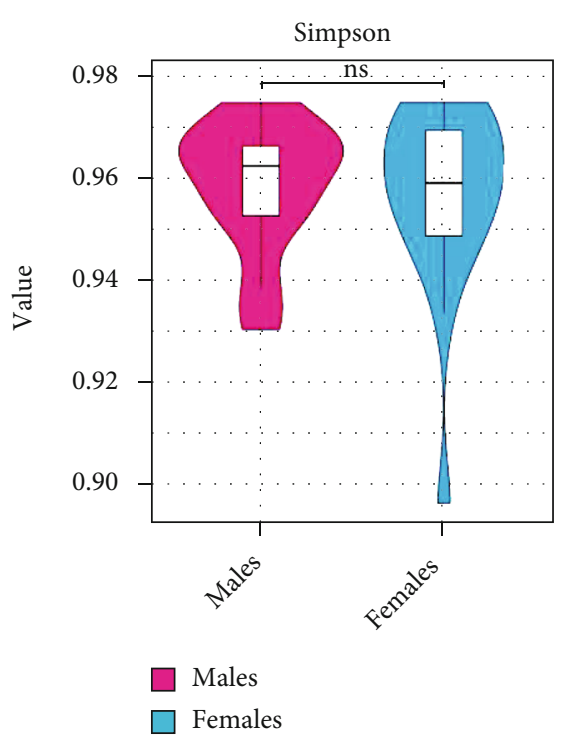

(e)

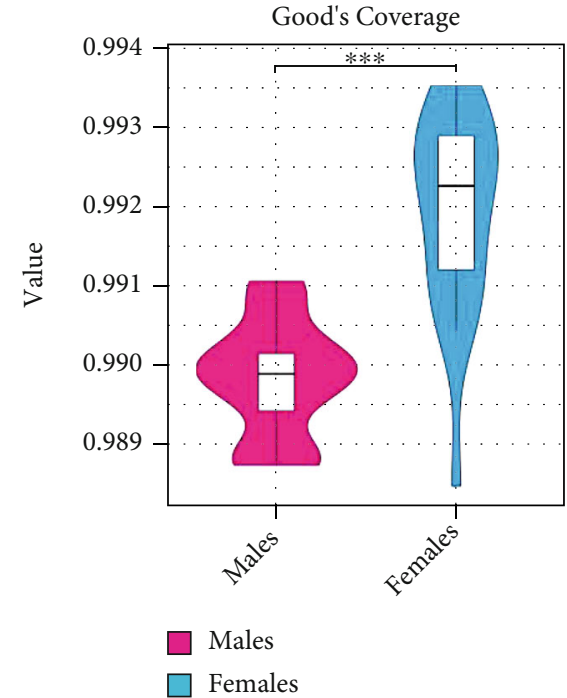

(f)

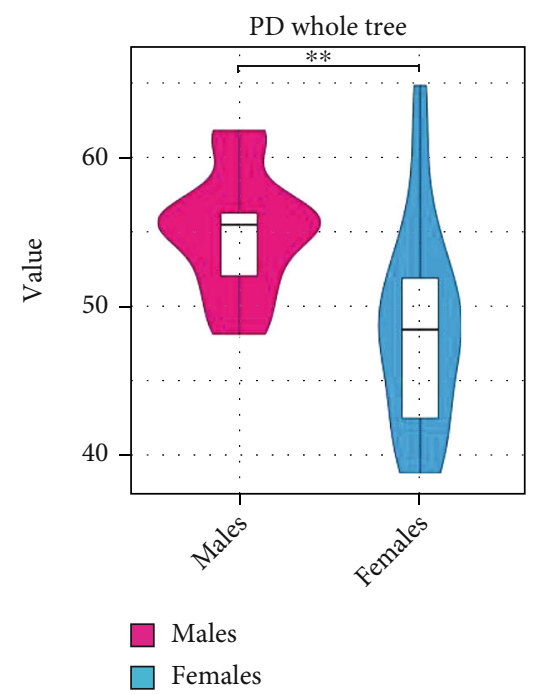

(g)

FIgURE 1: Venn diagram and $\alpha$-diversity analysis of elderly males and females with stage 1 periodontitis. (a) Venn diagram based on operational taxonomic units (OTUs). (b-g) Violin plots comparing $\alpha$-diversity indices (Chaol, observed species, Shannon index, Simpson index, Good's coverage, and PD whole tree) between males and females. ${ }^{* * *} P<0.001 ;{ }^{* *} P<0.01$; ns: not significant.

screen for potential biomarkers. As stated above, significant differences in the microbiota were observed between males and females with initial periodontitis (Figure 5(a)). A cladogram showing the most discriminative bacterial clades identified by LEfSe is shown in Figure 5(b). The following species were more predominant in the supragingival plaques of men than women: the class Alphaproteobacteria, its order SAR11_clade or Rhodobacterales, its family Clade_I/ Clade_II or Rhodobacteraceae, and its genus Clade_Ia/ Ambiguous_tax, along with the family Muribaculaceae/Bacteroidaceae and the genus Prevotellaceae_UCG_001/Prevotellaceae_NK3B31_group. In males, genus Haemophilus had the largest LDA score. However, these results show the significantly higher level of the phylum Epsilonbacteraeota and the corresponding class Campylobacteria, order Campy- lobacterales, family Campylobacteraceae, and genus Campylobacter, in the subgingival microbiotas of females. The microbiotas of women were also enriched with genera mainly belonging to the Bacteroidetes phylum, particularly the F082 and Bacteroidales_BS11_gut_group family. In addition, the phylum Actinobacteria and its genus Gardnerella, along with the phylum Firmicutes and its genus Selenomonas_1, were high in females. The phylum Proteobacteria and its genus Pelomonas were also high in females. Detailed data and statistics are provided in Table 2 .

3.4. Function Prediction. By linking the genomes to pathways via KEGG orthologue annotation, we determined differences in the estimated bacterial functional capabilities in the 12 males and 20 females and compared them using 


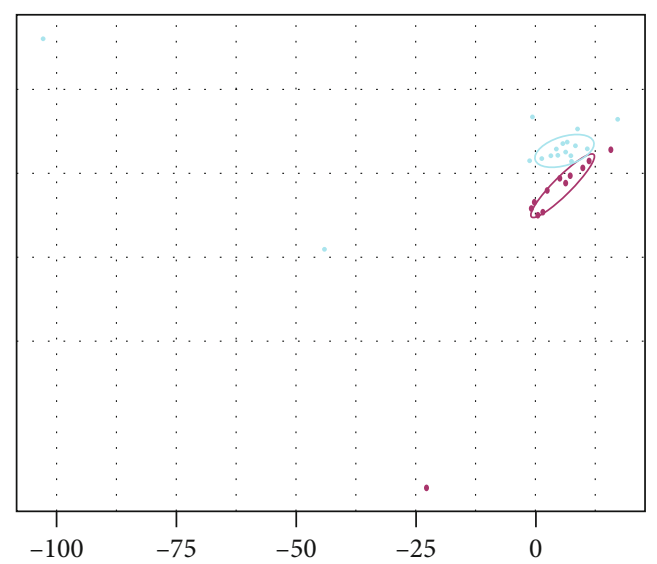

PC1 $8.16 \%$

Males

Females

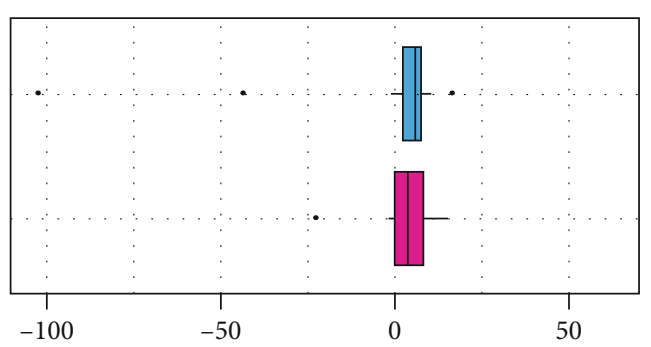

PC1 8.16\%

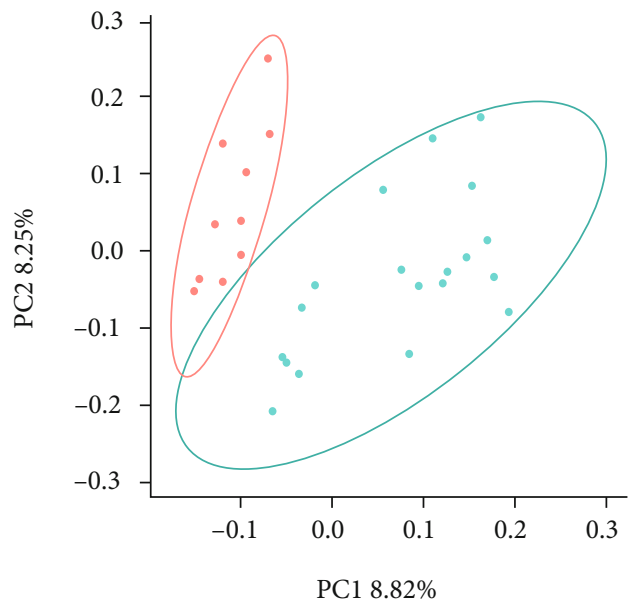

Males

Females

\section{Males}

Females

(a)

(b)

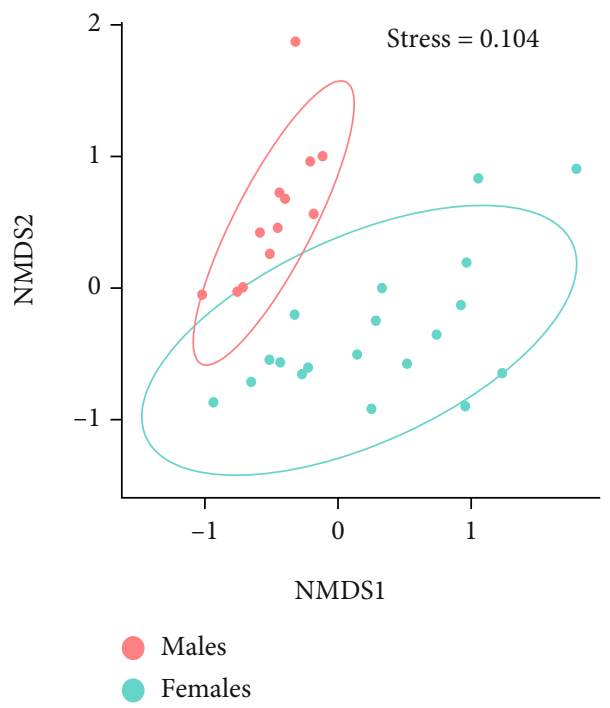

(c)

Figure 2: Similarity of microbial communities between elderly males and females with stage 1 periodontitis based on the unweighted UniFrac distance. (a) 2D diagram of principal component analysis (PCA). (b) 2D diagram of principal coordinate analysis. (c) 2D diagram of nonmetric multidimensional scale (NMDS) used to analyze the $\beta$-diversity of microbial communities between males and females. 


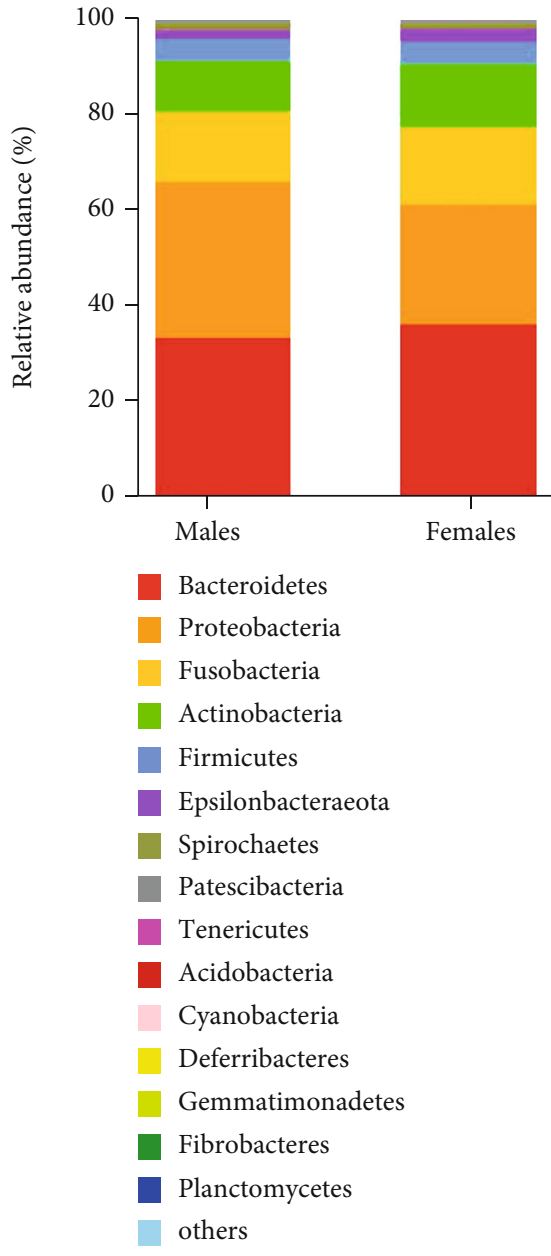

(a)

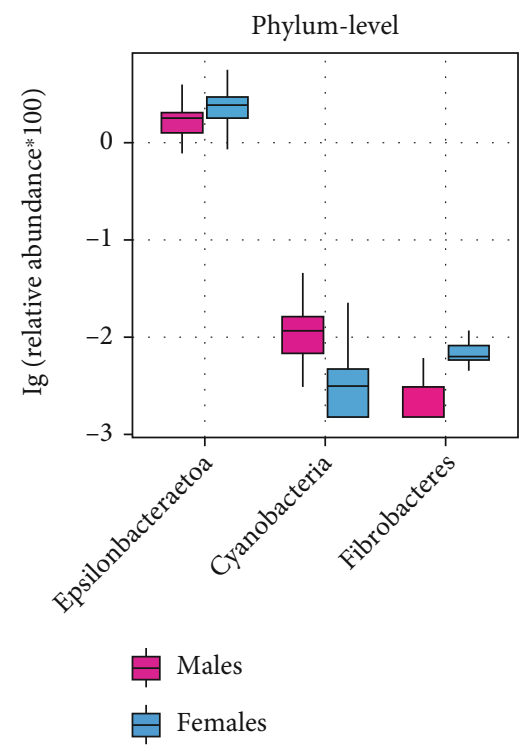

(c)

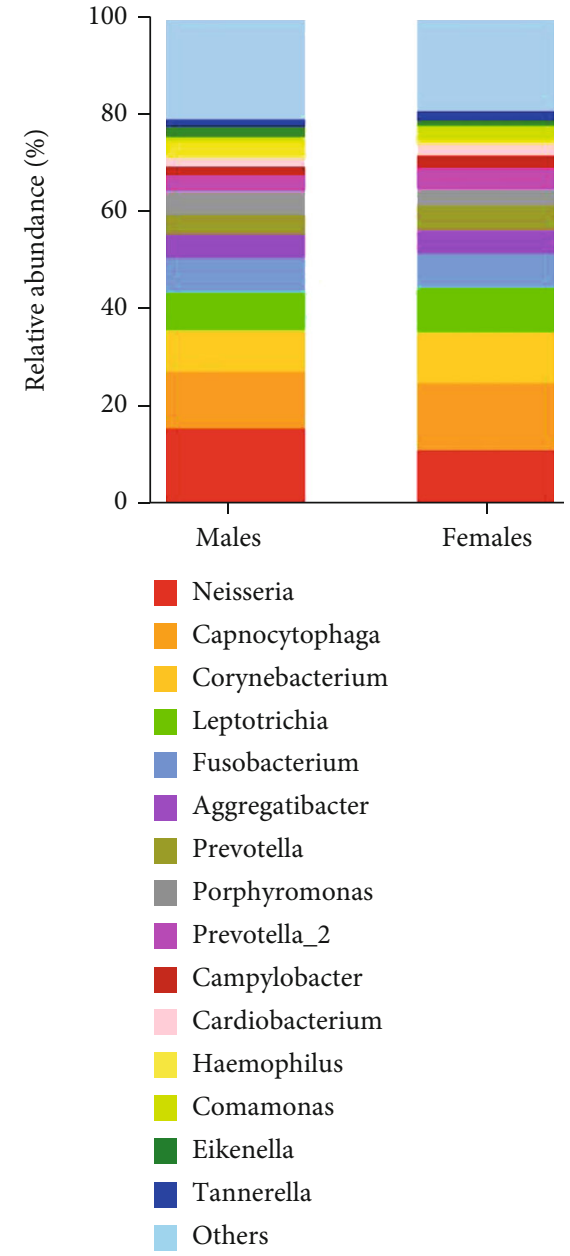

(b)

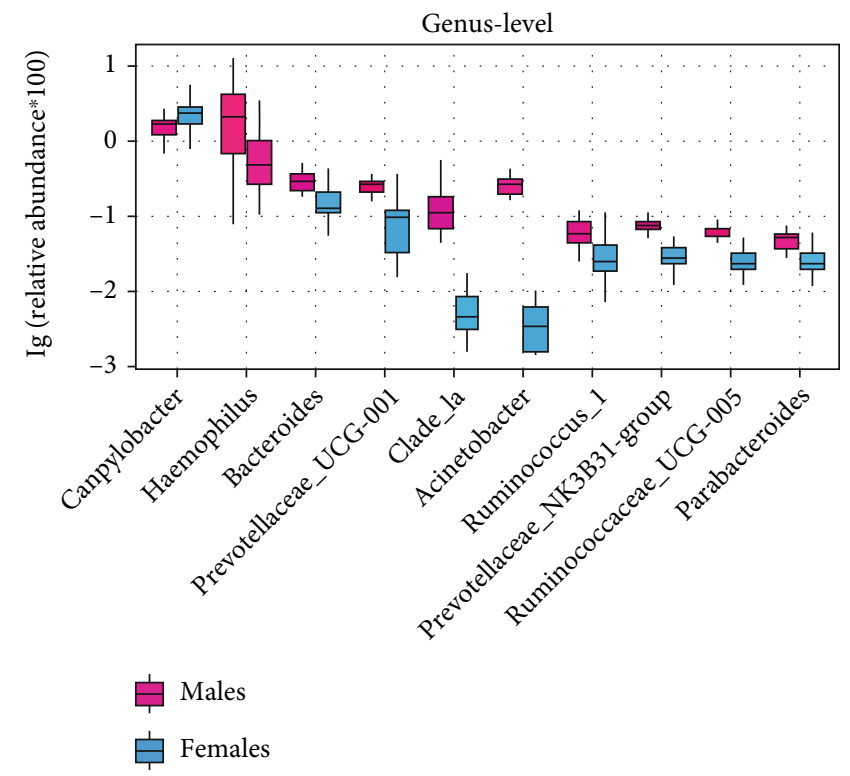

(d)

Figure 3: The oral microbiome composition of elderly males and females with stage 1 periodontitis. (a) Phylum level and (b) genus level composition. (c) Species difference analysis between males and females (group 2) by the Kruskal-Wallis test at the phylum and (d) genus levels. 


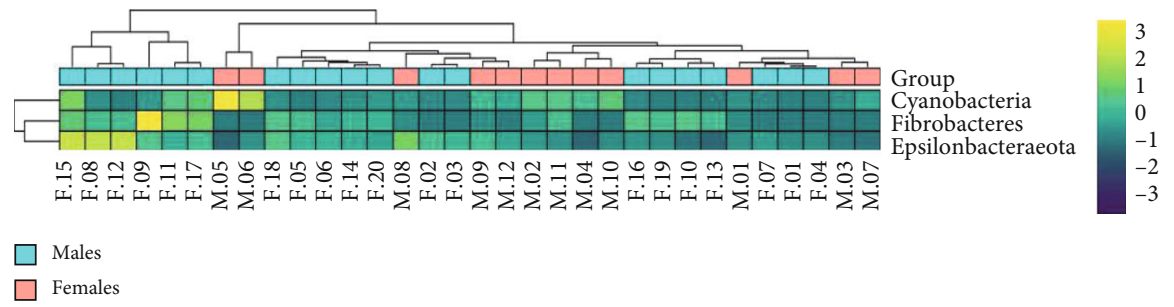

(a)

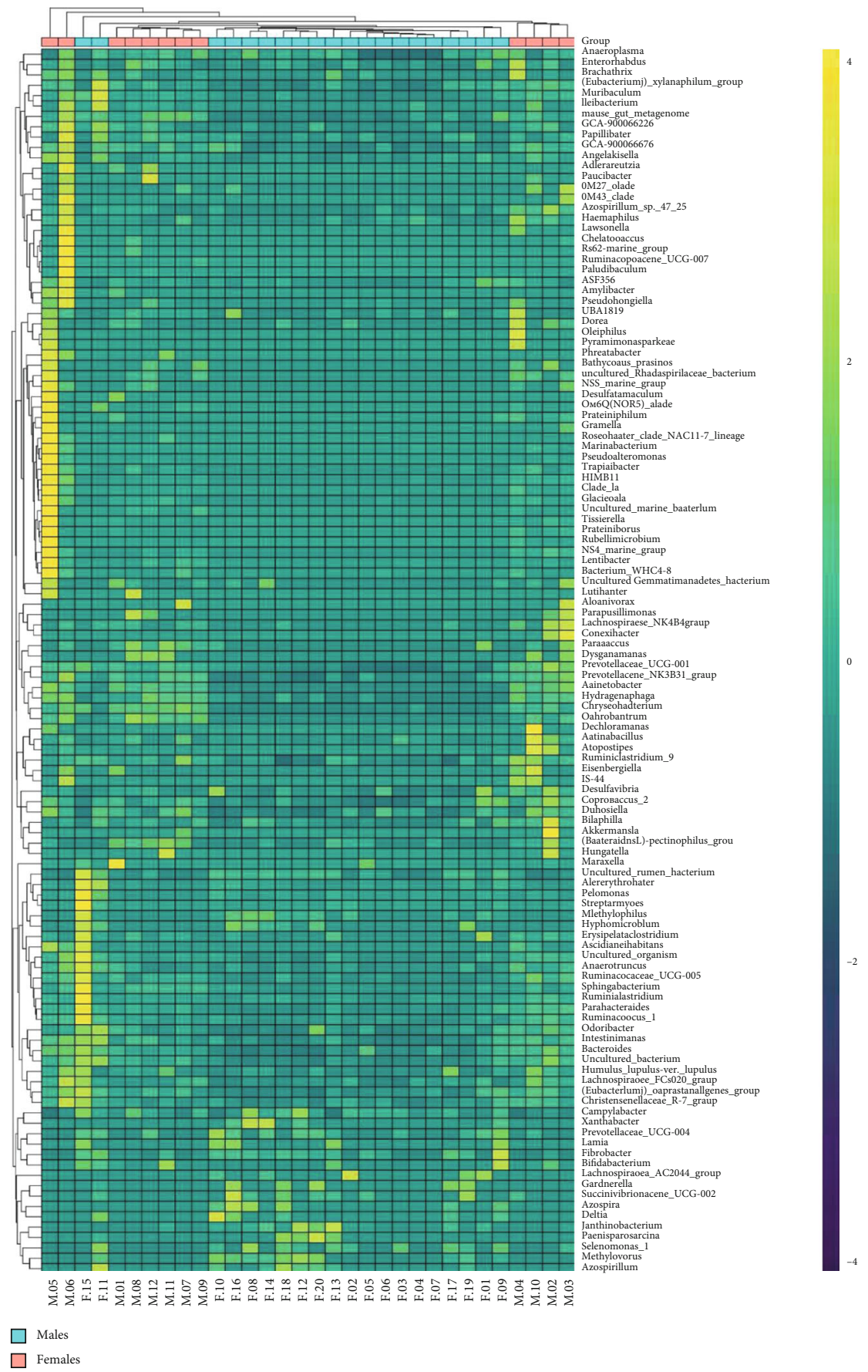

(b)

Figure 4: Heat map of differential oral microbiomes between elderly males and females with stage 1 periodontitis. (a) Phylum level. (b) Genus level. The sample information (group and number) and species annotation information are displayed on the horizontal axis and vertical axes, respectively. Colors indicate the Spearman rank correlation. 


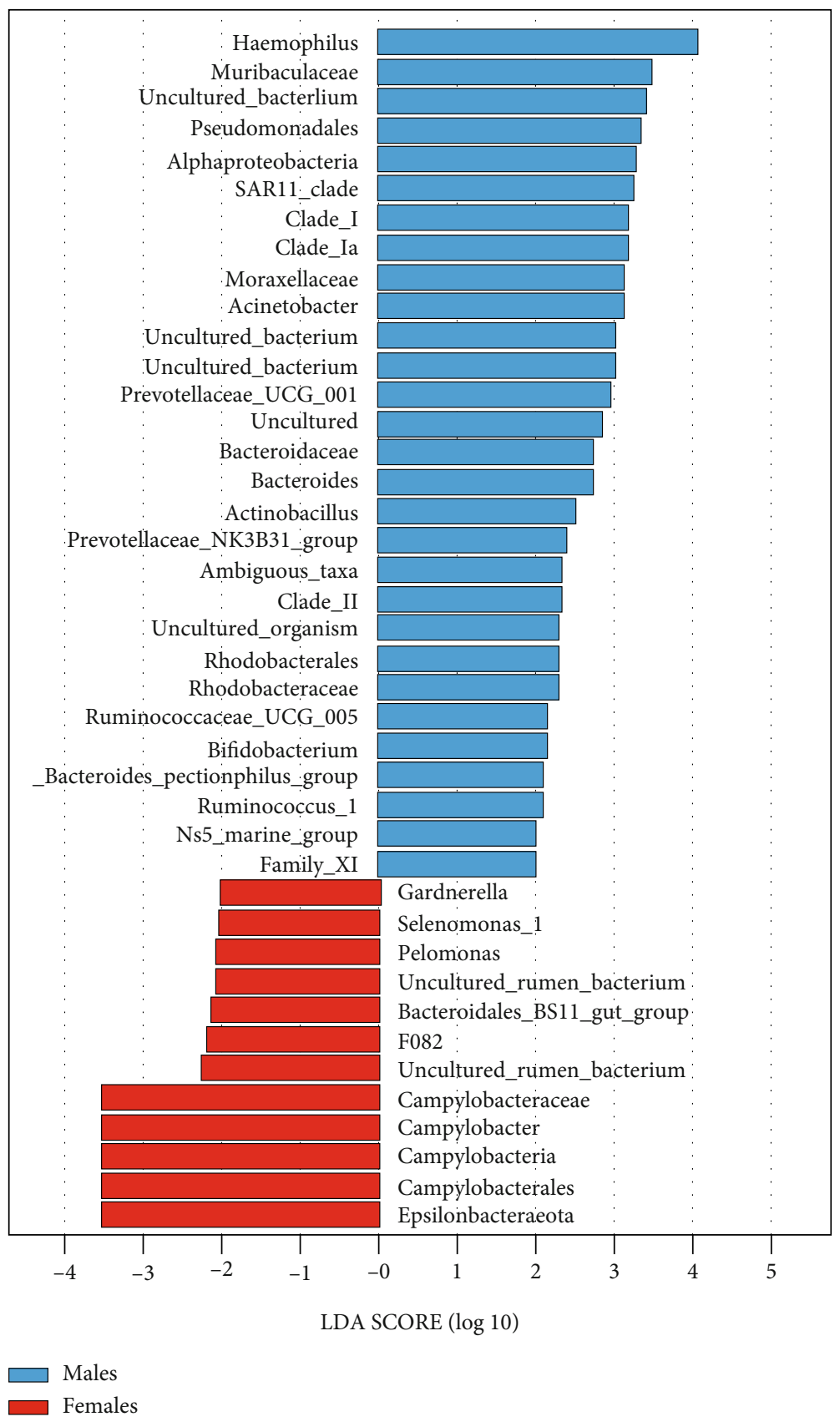

(a)

Figure 5: Continued. 


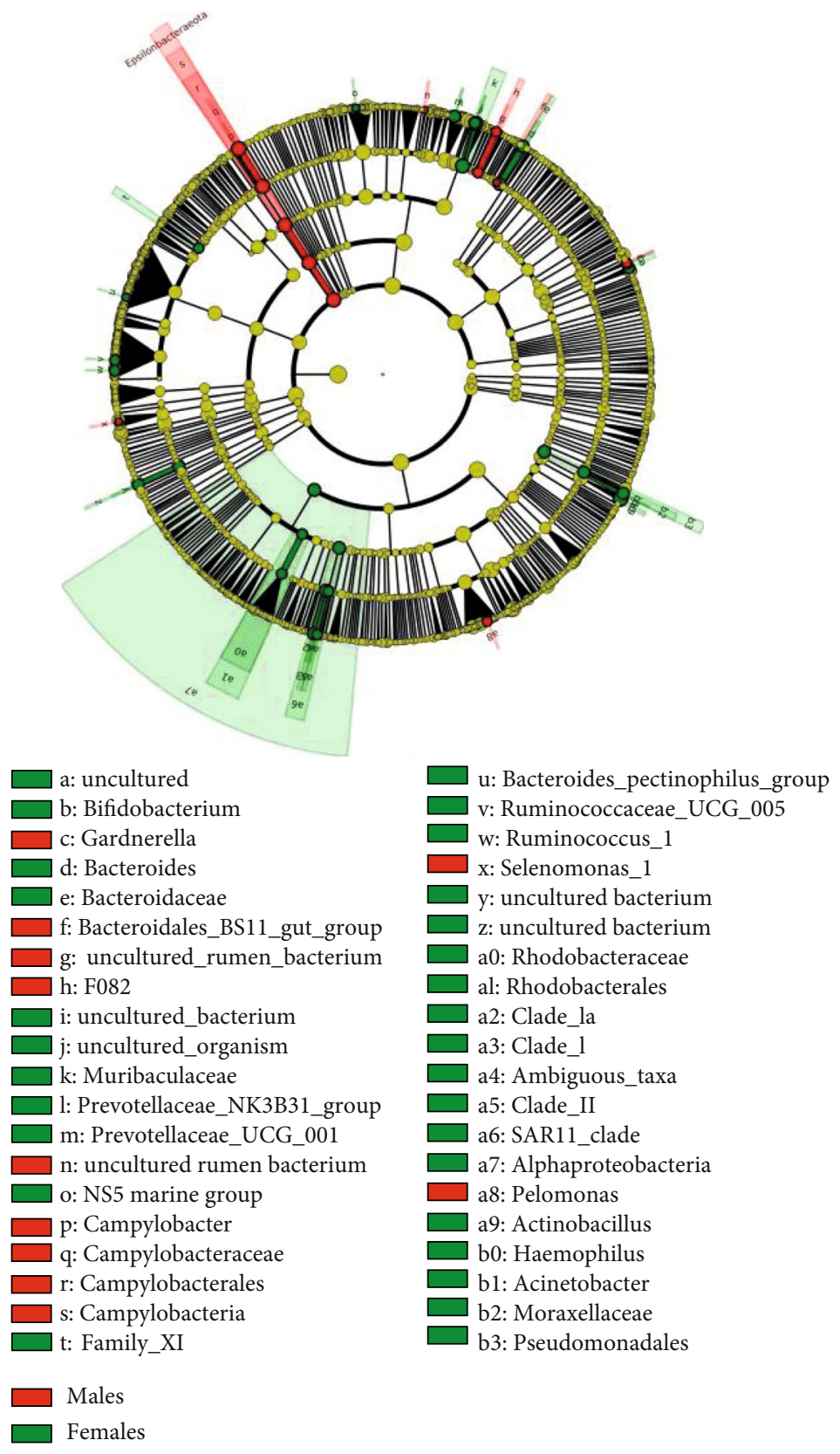

(b)

Figure 5: Different rich taxa between elderly males and females with stage 1 periodontitis analyzed by the linear discriminant analysis effect size. (a) Histogram of the LDA scores. (b) The phylogenetic tree in the form of a cladogram.

QIIME2 and PICRUSt2. At the phylum level, the relative proportions of functions associated with immune system diseases, neurodegenerative diseases, the circulatory system, and cardiovascular diseases appeared to increase in male patients. However, the functions associated with immune response, including the immune system, increased in females, whereas the immune-related functions decreased in males (Figure 6).

\section{Discussion}

In this study, we performed a detailed analysis of deep sequencing data and showed that the diversity and abun- dance of oral bacteria varies significantly between elderly male and female patients with initial periodontitis. The $\alpha$ diversity, reflecting species richness, was higher in males than females. The results of the $\beta$-diversity analysis showed that the samples were reasonably divided into different groups by gender. According to the relative abundance of species composition, LEfSe analysis showed that biomarkers in males were Haemophilus, family Muribaculaceae, and Clade_I/Clade_II, among others, whereas the dominant bacteria in females were Campylobacter, family F082, Bacteroidales_BS11_gut_group, Selenomonas_1, and Pelomonas. KEGG analysis showed that predicting changes in the female 
TABLE 2: Linear discriminant analysis (LDA) effect size (LEfSe) results of the distinct taxa between males and females.

\begin{tabular}{|c|c|c|}
\hline Biomarker & Groups & LDA value \\
\hline Bacteria.Proteobacteria.Gammaproteobacteria.Pasteurellales.Pasteurellaceae.Haemophilus & Males & 4.05 \\
\hline Bacteria.Bacteroidetes.Bacteroidia.Bacteroidales.Muribaculaceae & Males & 3.46 \\
\hline Bacteria.Bacteroidetes.Bacteroidia.Bacteroidales.Muribaculaceae.uncultured_bacterium & Males & 3.40 \\
\hline Bacteria.Proteobacteria.Alphaproteobacteria & Males & 3.28 \\
\hline Bacteria.Proteobacteria.Gammaproteobacteria.Pseudomonadales & Males & 3.33 \\
\hline Bacteria.Proteobacteria.Alphaproteobacteria.SAR11_clade & Males & 3.25 \\
\hline Bacteria.Proteobacteria.Alphaproteobacteria.SAR11_clade.Clade_I & Males & 3.18 \\
\hline Bacteria.Proteobacteria.Alphaproteobacteria.SAR11_clade.Clade_I.Clade_Ia & Males & 3.16 \\
\hline Bacteria.Bacteroidetes.Bacteroidia.Bacteroidales.Bacteroidaceae.Bacteroides & Males & 2.72 \\
\hline Bacteria.Bacteroidetes.Bacteroidia.Bacteroidales.Bacteroidaceae & Males & 2.72 \\
\hline Bacteria.Proteobacteria.Gammaproteobacteria.Pseudomonadales.Moraxellaceae & Males & 3.12 \\
\hline Bacteria.Bacteroidetes.Bacteroidia.Bacteroidales.Prevotellaceae.Prevotellaceae_UCG_001 & Males & 2.92 \\
\hline Bacteria.Proteobacteria.Gammaproteobacteria.Pseudomonadales.Moraxellaceae.Acinetobacter & Males & 3.10 \\
\hline Bacteria.Patescibacteria.Gracilibacteria.Absconditabacteriales_SR1_.uncultured_bacterium & Males & 3.02 \\
\hline Bacteria.Patescibacteria.Gracilibacteria.Absconditabacteriales_SR1_.uncultured_bacterium.uncultured_bacterium & Males & 3.02 \\
\hline Bacteria.Actinobacteria.Actinobacteria.Actinomycetales.Actinomycetaceae.Uncultured & Males & 2.84 \\
\hline Bacteria.Bacteroidetes.Bacteroidia.Bacteroidales.Muribaculaceae.uncultured_organism & Males & 2.27 \\
\hline Bacteria.Bacteroidetes.Bacteroidia.Bacteroidales.Prevotellaceae.Prevotellaceae_NK3B31_group & Males & 2.39 \\
\hline Bacteria.Proteobacteria.Gammaproteobacteria.Pasteurellales.Pasteurellaceae.Actinobacillus & Males & 2.51 \\
\hline Bacteria.Firmicutes.Clostridia.Clostridiales.Ruminococcaceae.Ruminococcus_1 & Males & 2.07 \\
\hline Bacteria.Firmicutes.Clostridia.Clostridiales.Ruminococcaceae.Ruminococcaceae_UCG_005 & Males & 2.14 \\
\hline Bacteria.Proteobacteria.Alphaproteobacteria.Rhodobacterales.Rhodobacteraceae & Males & 2.27 \\
\hline Bacteria.Proteobacteria.Alphaproteobacteria.Rhodobacterales & Males & 2.27 \\
\hline Bacteria.Proteobacteria.Alphaproteobacteria.SAR11_clade.Clade_II & Males & 2.31 \\
\hline Bacteria.Proteobacteria.Alphaproteobacteria.SAR11_clade.Clade_II.Ambiguous_taxa & Males & 2.32 \\
\hline Bacteria.Actinobacteria.Actinobacteria.Bifidobacteriales.Bifidobacteriaceae.Bifidobacterium & Males & 2.13 \\
\hline Bacteria.Firmicutes.Clostridia.Clostridiales.Family_XI & Males & 2.01 \\
\hline Bacteria.Firmicutes.Clostridia.Clostridiales.Lachnospiraceae._Bacteroides_pectinophilus_group & Males & 2.10 \\
\hline Bacteria.Bacteroidetes.Bacteroidia.Flavobacteriales.Flavobacteriaceae.NS5_marine_group & Males & 2.03 \\
\hline Bacteria.Epsilonbacteraeota & Females & 3.55 \\
\hline Bacteria.Epsilonbacteraeota.Campylobacteria.Campylobacterales & Females & 3.55 \\
\hline Bacteria.Epsilonbacteraeota.Campylobacteria & Females & 3.55 \\
\hline Bacteria.Epsilonbacteraeota.Campylobacteria.Campylobacterales.Campylobacteraceae.Campylobacter & Females & 3.53 \\
\hline Bacteria.Epsilonbacteraeota.Campylobacteria.Campylobacterales.Campylobacteraceae & Females & 3.53 \\
\hline Bacteria.Bacteroidetes.Bacteroidia.Bacteroidales.F082 & Females & 2.18 \\
\hline Bacteria.Bacteroidetes.Bacteroidia.Bacteroidales.F082.uncultured_rumen_bacterium & Females & 2.08 \\
\hline Bacteria.Proteobacteria.Gammaproteobacteria.Betaproteobacteriales.Burkholderiaceae.Pelomonas & Females & 2.06 \\
\hline Bacteria.Actinobacteria.Actinobacteria.Bifidobacteriales.Bifidobacteriaceae.Gardnerella & Females & 2.00 \\
\hline Bacteria.Bacteroidetes.Bacteroidia.Bacteroidales.Bacteroidales_BS11_gut_group & Females & 2.13 \\
\hline Bacteria.Firmicutes.Negativicutes.Selenomonadales.Veillonellaceae.Selenomonas_1 & Females & 2.00 \\
\hline Bacteria.Bacteroidetes.Bacteroidia.Bacteroidales.uncultured.uncultured_rumen_bacterium & Females & 2.25 \\
\hline
\end{tabular}

Only an LDA score of $>2.0$ is shown.

oral microbiota may be related to the immune system and immune system diseases are the main predictor of periodontitis in males.

Using the Kruskal-Wallis test for different species, the relative abundance of phyla Epsilonbacteraeota and Fibrobacteres and genus Campylobacter was higher in females than in males. The LEfSe analysis also identified Campylo- bacter as important. Campylobacter is Gram-negative microaerophilic bacteria that live as commensal organisms in the gastrointestinal tract. In addition to their own bacterial components, Campylobacter produces several different cytotoxins, including cytolethal distending toxin (CDT) and 1,3 galactosyltransferases involved in lipopolysaccharide (LPS) production, which play a role in colonization [29]. 


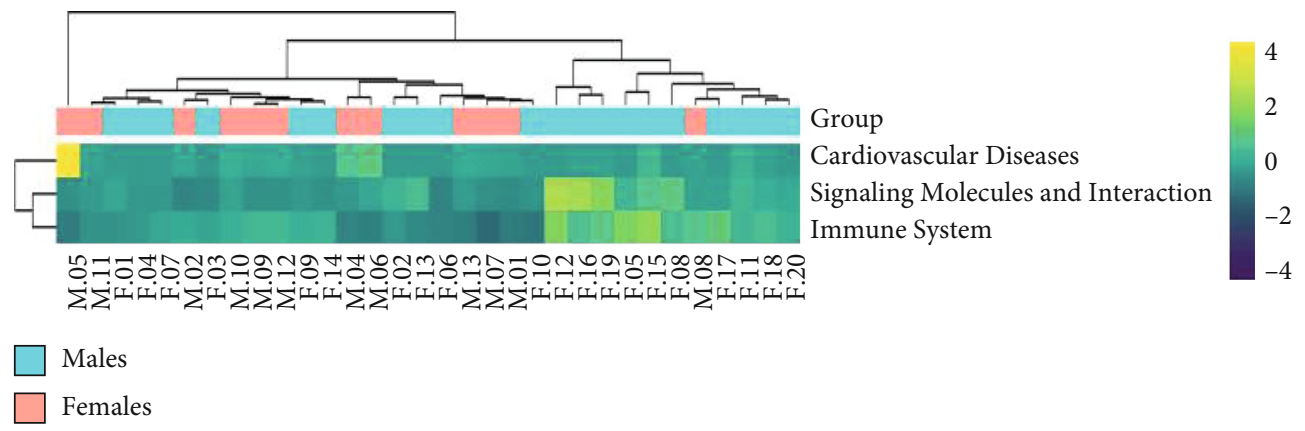

(a)

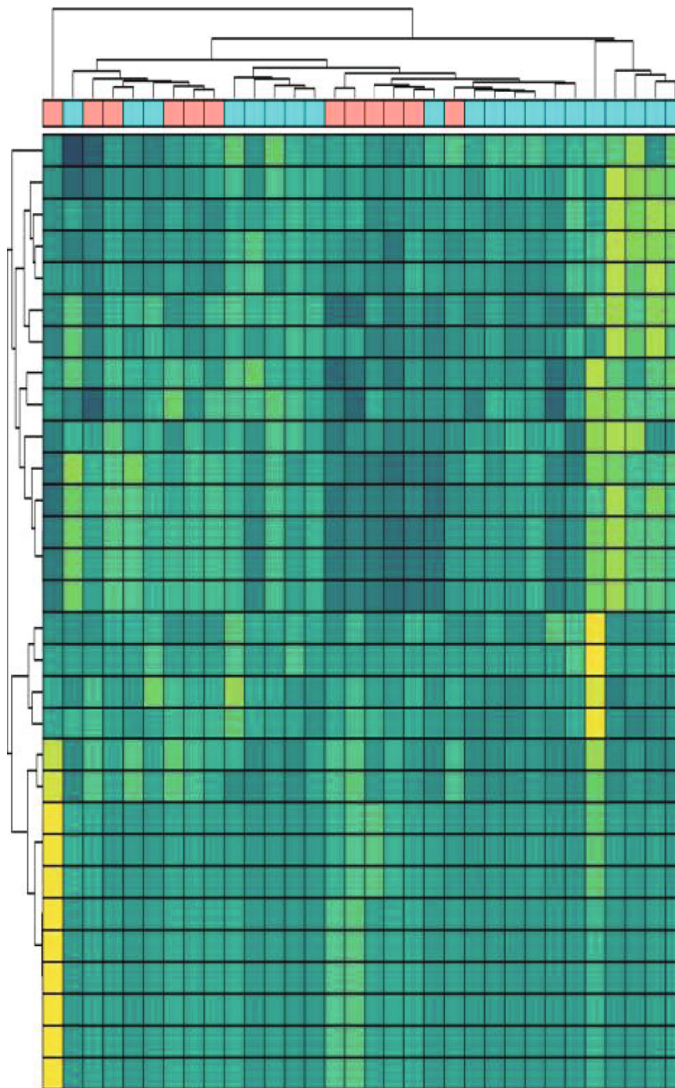

Group

Flavonoid biosynthesis

Stilbenoid, diarylheptanoid and gingerol biosynthesis

Cellular antigens

Ethylbenzene degradation

Adipocytokine signaling pathway

Bacterial toxins

Amoebiasis

Drug metabolism -other enzymes

Biosynthesis of ansamycins

1,1,1-Trichloro-2,2-bis(4-chlorophenyl)ethane (DDT) degradation NOD-like receptor signaling pathway

Protein processing in eridoplasmic reticulum

Prostate cancer

Progesterone-mediated oocyte maturation

Antigen processing and presentation

Biosynthesis of type Il polyketide backbone

Neuroactive ligand-receptor interaction

Secondary bile acid biosynthesis

Germination

Calcium signaling pathway

Photosynthesis -antennia proteins

Endocytosis

Fc gamma R-mediated phagocytosis

signaling pathway

p53 signaling pathway

Small cell lung cancer

Influenza A

Viral myocarditis

Colorectal cancer

Toxoplasmosis

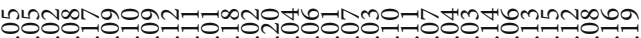

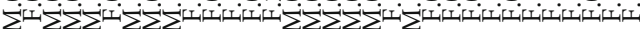

Males

Females

(b)

Figure 6: Continued. 


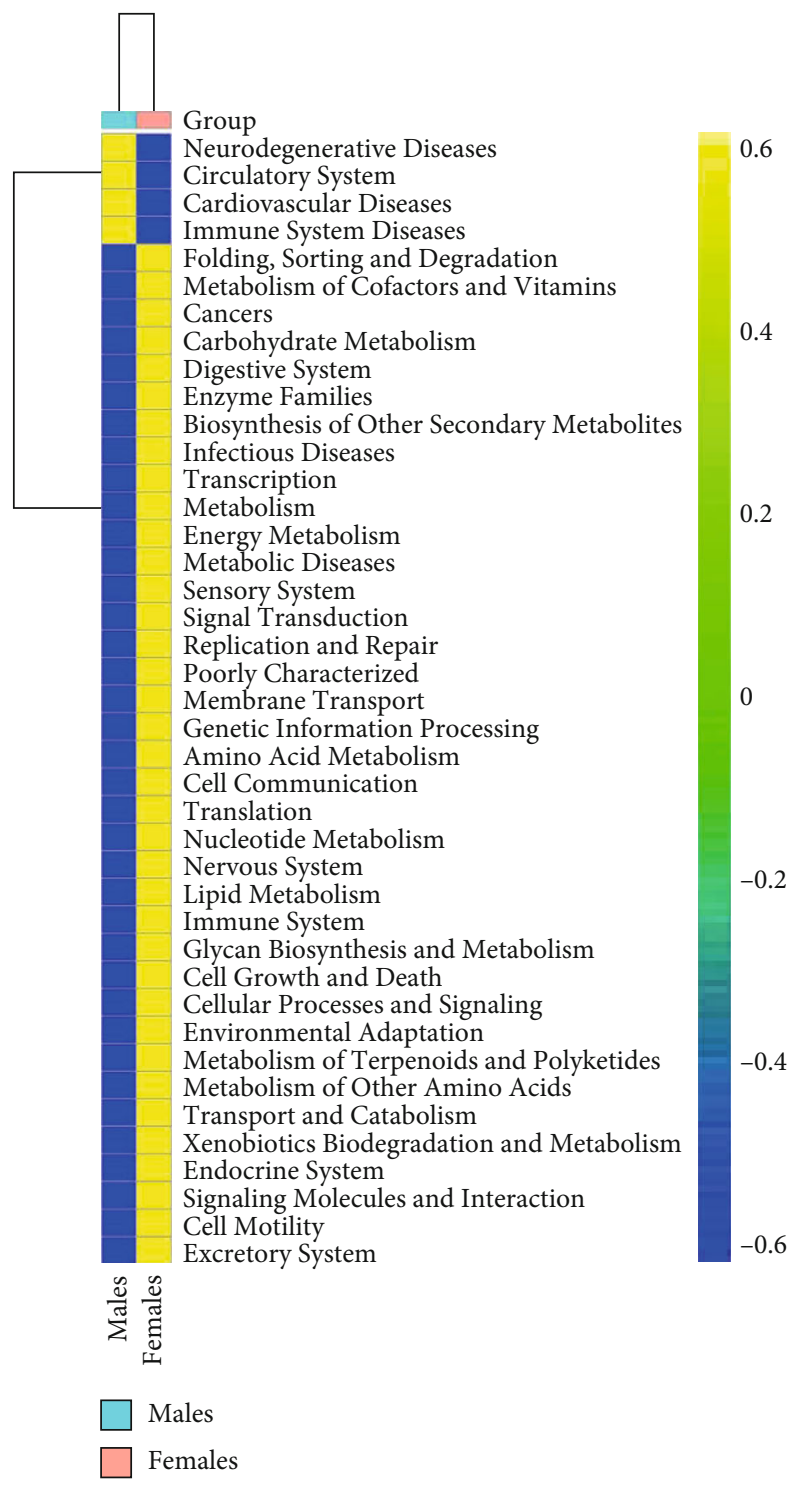

(c)

FiguRE 6: KEGG pathway enrichment analysis. (a) The different pathways in individual patients are shown in clustering heat maps at level 2 and (b) level 3 of KEGG. (c) At level 2 of KEGG, the different pathways between the two groups were clustered into a heat map.

Lundmark et al. found that Campylobacter conisus is more abundant in healthy individuals through $16 \mathrm{~S}$ rRNA sequencing of saliva samples from patients with chronic periodontitis and healthy periodontal controls [30]. An observational cross-sectional study of 76 postmenopausal women found Campylobacter rectus in the oral microflora of these subjects by real-time PCR [31]. Our results also showed that Campylobacter was abundant in the postmenopausal women compared with elderly men. Thus, the increase in Campylobacter may play a potential positive role in periodontal health in postmenopausal women. Interestingly, a previous study investigated the composition of the tongue microflora in 16 intraoral halitosis (IOH) patients and 10 healthy subjects and found that Campylobacter was significantly abundant in the IOH group [32]. This difference may be due to age, hormone levels, noncomparable study populations, and the diverse mechanisms of oral diseases.
An important factor affecting the oral microbiome is age [33]. The outer membrane vesicles or gingipains of $P$. gingivalis and free soluble bacterial components of A. actinomycetemcomitans released into the circulation can induce a proatherogenic responses in endothelial cells [34], suggesting that the microbiota plays a role in cardiovascular disease in older adults. This may be associated with a general decrease in immune function and the development of chronic inflammation during aging. In men, age correlates with a more pronounced decrease in the total number of $\mathrm{T}$ and $\mathrm{B}$ cells and larger increase in senescent CD8+T effector memory cells compared to women $[35,36]$. In contrast, menopause is associated with increased levels of IL- $1 \beta$, IL6 , and TNF- $\alpha$ (proinflammatory) and reduced levels of IFN- $\gamma$ (anti-inflammatory). In aged women, monocytes have a proinflammatory phenotype and NK cell robust cytotoxic activity [37]. Furthermore, periodontitis has been reported 
to occur more often in postmenopausal women who do not receive hormone replacement compared to premenopausal women [31]. Our results showed that Haemophilus had the largest LDA score in males. Haemophilus is a Gramnegative bacteria that can only grow with fresh blood during artificial culture. It is deposited mainly in the throat and oral mucosa of humans and animals, which could cause primary suppurative infection and serious secondary infection [38]. The main virulence factors include capsule and lipooligosaccharide (LOS). The LOS of Haemophilus ducreyi induces immunosuppressive enzyme expression in DCs, largely through type I IFN- and TNF- $\alpha$-dependent mechanisms, as well as the MAPK, NF- $\kappa$ B, and JAK-STAT pathways [39]. Other studies have shown that Haemophilus is related to sex differences. A $16 \mathrm{~S}$ rRNA gene sequencing analysis of sputum samples from patients with asthma and normal controls found that Haemophilus spp. are associated with asthma in men but not in women [40]. On the other hand, in contrast to the present study, Raju et al. found a high relative abundance of Haemophilus in the saliva microbiota of females [41]. The LEfSe analysis identified additional biomarkers, including Rhodobacteraceae, Actinobacillus, Prevotellaceae, Muribaculaceae, and Bacteroidaceae. The immunomodulatory effects of LPS from Rhodopseudomonas sphaeroides are mainly the result of eliminating the inhibitory effects of T cells; this permitted the positive effects of amplifier $\mathrm{T}$ cells to be more fully expressed, resulting in an increased antibody response [42]. Furthermore, Actinobacillus actinomycetemcomitans, one of the major causative agents of chronic inflammatory periodontal disease, has been shown to cause a specific immune response by the host [43].

The dominant bacteria mentioned above may change the host immune response. We used KEGG to predict signaling pathways involved by the bacteria. Immune system diseases, neurodegenerative diseases, circulatory system diseases, and cardiovascular diseases were enriched in males, whereas immune system diseases, infectious diseases, metabolic diseases, and endocrine system diseases were increased in females. Jansen et al. have indicated that female-biased Gene Ontology categories are highly enriched for various immune system functions, including the TLR3 and TLR4 pathways, as well as genes linked to autoimmune diseases and genes regulated by estrogen and LPS [44]. As mentioned above, Campylobacter is a commensal organism in the gastrointestinal tract. Commensal microorganisms are greatly involved in maintaining homeostasis and health not only by blocking microbial activity but also by reinforcing the human immune system via specialized mechanisms [45], which further indicates a correlation between the oral microbiome and immune system. In KEGG pathway analysis, the endocrine system was enriched in females. The differences may be related to the change in female sexual hormones throughout life, one of the factors that plays an essential role in microbiota modulation. With increasing age, estrogen and androgen levels vary in both males and females with some commonality, as well as significant differences [46]. Though estrogen levels drastically decrease with menopause in women, androgens progressively decrease in both sexes starting at approximately 30 years of age [47-49]. Changes in periodontal status have also been found to be associated with variations in levels of sex hormones [50]. Finally, specific bacterial species, such as $P$. gingivalis and Tannerella forsythensis, have been shown to be important in postmenopausal women regarding the etiology of periodontitis [51].

\section{Conclusion}

Overall, our findings indicate that gender may be a differentiating factor in the microbial composition of the subgingival plaques of elderly patients with initial periodontitis. Future oral microbiome studies may yield better resolution if the context of sex-specific differences is considered.

\section{Data Availability}

Raw reads have been deposited at NCBI under the BioProject accession number PRJNA763744.

\section{Conflicts of Interest}

The authors declare that there are no conflicts of interest regarding the publication of this paper.

\section{Authors' Contributions}

Jie Zhao and Ying-Hui Zhou contributed equally to this manuscript.

\section{Acknowledgments}

We thank all of the subgingival plaque donors who participated in our study. This study was supported by the National Natural Science Foundation of China (Grants 81773339,81800788 , and 82001004), the Science and Technology Department of Hunan Province, China (Grants 2017WK2041 and 2018SK52511), the National Natural Science Foundation of Hunan Province (Grant 2018JJ3708), the fund for Xiangya Clinical Medicine Database of Central South University (Grant 2014-ZDYZ-1-16), and the Open Sharing Fund for the Large-scale Instruments and Equipment of Central South University.

\section{References}

[1] M. Zorba, A. Melidou, A. Patsatsi, E. Ioannou, and A. Kolokotronis, "The possible role of oral microbiome in autoimmunity," International Journal of Women's Dermatology, vol. 6, no. 5, pp. 357-364, 2020.

[2] E. Xiao, M. Mattos, G. H. A. Vieira et al., "Diabetes enhances IL-17 expression and alters the oral microbiome to increase its pathogenicity," Cell Host Microbe, vol. 22, no. 1, pp. 120 128.e4, 2017.

[3] H. Mohammed and E. M. Varoni, "Oral dysbiosis in pancreatic cancer and liver cirrhosis: a review of the literature," Biomedicines, vol. 6, no. 4, p. 115, 2018.

[4] M. Dioguardi, V. Crincoli, L. Laino et al., "The role of periodontitis and periodontal bacteria in the onset and progression of Alzheimer's disease: a systematic review," Journal of Clinical Medicine, vol. 9, no. 2, p. 495, 2020. 
[5] P. M. Bartold and I. Lopez-Oliva, "Periodontitis and rheumatoid arthritis: an update 2012-2017," Periodontology 2000, vol. 83, no. 1, pp. 189-212, 2020.

[6] B. Sampaio-Maia, I. M. Caldas, M. L. Pereira, D. PérezMongiovi, and R. Araujo, "The oral microbiome in health and its implication in oral and systemic diseases," Advances in Applied Microbiology, vol. 97, pp. 171-210, 2016.

[7] X. Fan, A. V. Alekseyenko, J. Wu et al., "Human oral microbiome and prospective risk for pancreatic cancer: a population-based nested case-control study," Gut, vol. 67, no. 1, pp. 120-127, 2018.

[8] S. S. Dominy, C. Lynch, F. Ermini et al., "Porphyromonas gingivalis in Alzheimer's disease brains: evidence for disease causation and treatment with small-molecule inhibitors," Science Advances, vol. 5, no. 1, article eaau3333, 2019.

[9] K. Bunte and T. Beikler, "Th17 cells and the IL-23/IL-17 axis in the pathogenesis of periodontitis and immune-mediated inflammatory diseases," International Journal of Molecular Sciences, vol. 20, no. 14, p. 3394, 2019.

[10] B. Flemer, R. D. Warren, M. P. Barrett et al., "The oral microbiota in colorectal cancer is distinctive and predictive," Gut, vol. 67, no. 8, pp. 1454-1463, 2018.

[11] J. P. Rodenburg, A. J. Winkelhoff, E. G. Winkel, R. J. Goene, F. Abbas, and J. Graaff, "Occurrence of Bacteroides gingivalis, Bacteroides intermedius and Actinobacillus actinomycetemcomitans in severe periodontitis in relation to age and treatment history," Journal of Clinical Periodontology, vol. 17, no. 6, pp. 392-399, 1990.

[12] J. M. Albandar, "Global risk factors and risk indicators for periodontal diseases," Periodontology 2000, vol. 29, pp. 177206, 2002.

[13] R. Lira-Junior, S. Åkerman, B. Klinge, E. A. Boström, and A. Gustafsson, "Salivary microbial profiles in relation to age, periodontal, and systemic diseases," PLoS One, vol. 13, no. 3, article e0189374, 2018.

[14] J. M. Albandar, "Periodontal diseases in North America," Periodontology 2000, vol. 29, pp. 31-69, 2002.

[15] M. Ferraro and A. R. Vieira, "Explaining gender differences in caries: a multifactorial approach to a multifactorial disease," International Journal of Dentistry, vol. 2010, Article ID 649643, 2010.

[16] D. K. Dennison and T. E. Van Dyke, "The acute inflammatory response and the role of phagocytic cells in periodontal health and disease," Periodontology 2000, vol. 14, pp. 54-78, 1997.

[17] K. Yamazaki, H. Yoshie, and G. J. Seymour, "T cell regulation of the immune response to infection in periodontal diseases," Histology and Histopathology, vol. 18, no. 3, pp. 889-896, 2003.

[18] T. Berglundh and M. Donati, "Aspects of adaptive host response in periodontitis," Journal of Clinical Periodontology, vol. 32, Suppl 6, pp. 87-107, 2005.

[19] H. Youssef and P. Stashenko, "Interleukin-1 and estrogen protect against disseminating dentoalveolar infections," International Journal of Oral Science, vol. 9, no. 1, pp. 1623, 2017.

[20] A. Trigunaite, J. Dimo, and T. N. Jørgensen, "Suppressive effects of androgens on the immune system," Cellular Immunology, vol. 294, no. 2, pp. 87-94, 2015.

[21] J. A. Rettew, Y. M. Huet-Hudson, and I. Marriott, “Testosterone reduces macrophage expression in the mouse of toll-like receptor 4, a trigger for inflammation and innate immunity," Biology of Reproduction, vol. 78, no. 3, pp. 432-437, 2008.
[22] G. D’Souza, T. S. McNeel, and C. Fakhry, "Understanding personal risk of oropharyngeal cancer: risk-groups for oncogenic oral HPV infection and oropharyngeal cancer," Annals of Oncology, vol. 28, no. 12, pp. 3065-3069, 2017.

[23] J. Y. An, R. Darveau, and M. Kaeberlein, "Oral health in geroscience: animal models and the aging oral cavity," Geroscience, vol. 40, no. 1, pp. 1-10, 2018.

[24] P. M. Preshaw, K. Henne, J. J. Taylor, R. A. Valentine, and G. Conrads, "Age-related changes in immune function (immune senescence) in caries and periodontal diseases: a systematic review," Journal of Clinical Periodontology, vol. 44, Supplement 18, pp. S153-S177, 2017.

[25] M. S. Tonetti, H. Greenwell, and K. S. Kornman, "Staging and grading of periodontitis: framework and proposal of a new classification and case definition," Journal of Clinical Periodontology, vol. 45, Suppl 20, pp. S149-S161, 2018.

[26] T. Rognes, T. Flouri, B. Nichols, C. Quince, and F. Mahé, "VSEARCH: a versatile open source tool for metagenomics," PeerJ, vol. 4, article e2584, 2016.

[27] Q. Wang, G. M. Garrity, J. M. Tiedje, and J. R. Cole, "Naive Bayesian classifier for rapid assignment of rRNA sequences into the new bacterial taxonomy," Applied and Environmental Microbiology, vol. 73, no. 16, pp. 5261-5267, 2007.

[28] M. G. Langille, J. Zaneveld, J. G. Caporaso et al., "Predictive functional profiling of microbial communities using $16 \mathrm{~S}$ rRNA marker gene sequences," Nature Biotechnology, vol. 31, no. 9, pp. 814-821, 2013.

[29] C. Fitzgerald, "Campylobacter," Clinics in Laboratory Medicine, vol. 35, no. 2, pp. 289-298, 2015.

[30] A. Lundmark, Y. O. O. Hu, M. Huss, G. Johannsen, A. F. Andersson, and T. Yucel-Lindberg, "Identification of salivary microbiota and its association with host inflammatory mediators in periodontitis," Frontiers in Cellular and Infection Microbiology, vol. 9, p. 216, 2019.

[31] S. Hernández-Vigueras, B. Martínez-Garriga, M. C. Sánchez et al., "Oral microbiota, periodontal status, and osteoporosis in postmenopausal females," Journal of Periodontology, vol. 87, no. 2, pp. 124-133, 2016.

[32] K. Seerangaiyan, A. J. van Winkelhoff, H. J. M. Harmsen, J. W. A. Rossen, and E. G. Winkel, "The tongue microbiome in healthy subjects and patients with intra-oral halitosis," Journal of Breath Research, vol. 11, no. 3, article 036010, 2017.

[33] G. R. Persson, "Dental geriatrics and periodontitis," Periodontology 2000, vol. 74, no. 1, pp. 102-115, 2017.

[34] L. Reyes, D. Herrera, E. Kozarov, S. Roldán, and A. ProgulskeFox, "Periodontal bacterial invasion and infection: contribution to atherosclerotic pathology," Journal of Clinical Periodontology, vol. 40, Suppl 14, pp. S30-S50, 2013.

[35] J. Yan, J. M. Greer, R. Hull et al., "The effect of ageing on human lymphocyte subsets: comparison of males and females," Immunity \& Ageing, vol. 7, no. 1, p. 4, 2010.

[36] K. Hirokawa, M. Utsuyama, Y. Hayashi, M. Kitagawa, T. Makinodan, and T. Fulop, "Slower immune system aging in women versus men in the Japanese population," Immunity \& Ageing, vol. 10, no. 1, p. 19, 2013.

[37] M. R. Gubbels Bupp, T. Potluri, A. L. Fink, and S. L. Klein, "The confluence of sex hormones and aging on immunity," Frontiers in Immunology, vol. 9, p. 1269, 2018.

[38] N. Mihancea, "Haemophilus infections in humans," Roumanian Archives of Microbiology and Immunology, vol. 52, no. 1, pp. 5-13, 1993. 
[39] W. Li, B. P. Katz, and S. M. Spinola, "Haemophilus ducreyi lipooligosaccharides induce expression of the immunosuppressive enzyme indoleamine 2,3-dioxygenase via type I interferons and tumor necrosis factor alpha in human dendritic cells," Infection and Immunity, vol. 79, no. 8, pp. 3338-3347, 2011.

[40] R. Chen, L. Wang, T. Koch et al., "Sex effects in the association between airway microbiome and asthma," Annals of Allergy, Asthma \& Immunology, vol. 125, no. 6, pp. 652-657.e3, 2020.

[41] S. C. Raju, S. Lagström, P. Ellonen et al., "Gender-specific associations between saliva microbiota and body size," Frontiers in Microbiology, vol. 10, p. 767, 2019.

[42] P. J. Baker, C. E. Taylor, P. W. Stashak et al., "Inactivation of suppressor $\mathrm{T}$ cell activity by the nontoxic lipopolysaccharide of Rhodopseudomonas sphaeroides," Infection and Immunity, vol. 58, no. 9, pp. 2862-2868, 1990.

[43] H. H. Zadeh, F. C. Nichols, and K. T. Miyasaki, "The role of the cell-mediated immune response to Actinobacillus actinomycetemcomitans and Porphyromonas gingivalis in periodontitis," Periodontology 2000, vol. 20, pp. 239-288, 1999.

[44] R. Jansen, S. Batista, A. I. Brooks et al., "Sex differences in the human peripheral blood transcriptome," BMC Genomics, vol. 15, no. 1, p. 33, 2014.

[45] A. L. Kau, P. P. Ahern, N. W. Griffin, A. L. Goodman, and J. I. Gordon, "Human nutrition, the gut microbiome and the immune system," Nature, vol. 474, no. 7351, pp. 327-336, 2011.

[46] B. C. Özdemir and G. P. Dotto, "Sex hormones and anticancer immunity," Clinical Cancer Research, vol. 25, no. 15, pp. 46034610, 2019.

[47] C. Castelo-Branco and I. Soveral, "The immune system and aging: a review," Gynecological Endocrinology, vol. 30, no. 1, pp. 16-22, 2014.

[48] S. L. Davison, R. Bell, S. Donath, J. G. Montalto, and S. R. Davis, "Androgen levels in adult females: changes with age, menopause, and oophorectomy," The Journal of Clinical Endocrinology and Metabolism, vol. 90, no. 7, pp. 3847-3853, 2005.

[49] S. M. Harman, E. J. Metter, J. D. Tobin, J. Pearson, M. R. Blackman, and Baltimore Longitudinal Study of Aging, "Longitudinal effects of aging on serum total and free testosterone levels in healthy men. Baltimore longitudinal study of aging," The Journal of Clinical Endocrinology and Metabolism, vol. 86, no. 2, pp. 724-731, 2001.

[50] P. Mascarenhas, R. Gapski, K. al-Shammari, and H. L. Wang, "Influence of sex hormones on the periodontium," Journal of Clinical Periodontology, vol. 30, no. 8, pp. 671-681, 2003.

[51] R. M. Brennan, R. J. Genco, K. M. Hovey, M. Trevisan, and J. Wactawski-Wende, "Clinical attachment loss, systemic bone density, and subgingival calculus in postmenopausal women," Journal of Periodontology, vol. 78, no. 11, pp. 2104-2111, 2007. 